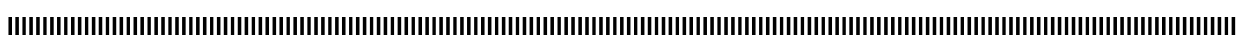

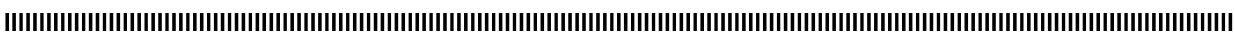

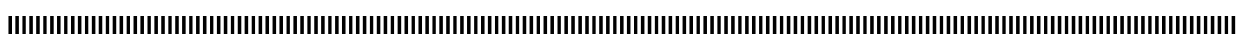

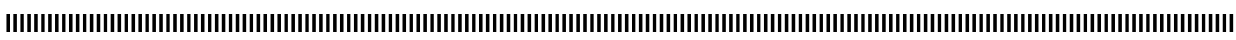

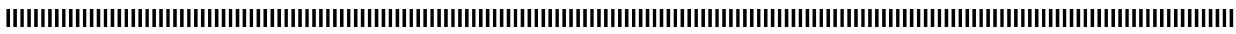

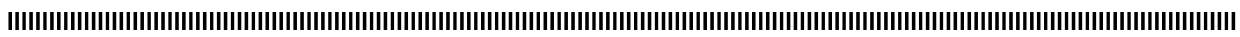

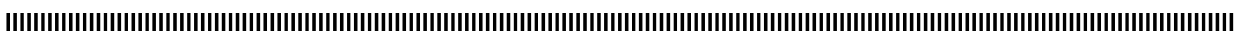

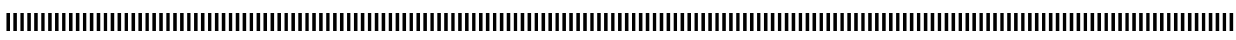

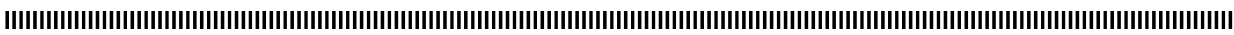

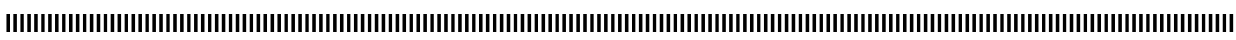

\title{
Un modèle Darcy-Forchheimer pour un écoulement dans un milieu poreux fracturé
}

\author{
Najla Frih *, Jean E. Roberts **, Ali Saada *** \\ * N. Frih ENIT-LAMSIN, BP 37, 1002 Tunis-le Belvédère, Tunisie, \\ najla.frih@lamsin.rnu.tn \\ ** J. E. Roberts, INRIA-Rocquencourt, B.P 105, 78153 Le Chesnay Cedex, France, \\ jean.roberts@inria.fr \\ *** A. Saada, ENIT-LAMSIN, BP 37, 1002 Tunis-le Belvédère, Tunisie, \\ ali.saada@ipein.rnu.tn
}

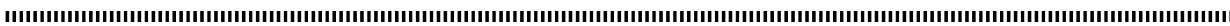

RÉSUMÉ. Nous proposons un modèle numérique d'un écoulement monophasique d'un fluide incompressible dans un milieu poreux fracturé. Les lois d'écoulement sont celle de Forchheimer dans la fracture et de Darcy dans la matrice rocheuse.

ABSTRACT. We propose a numerical model for the flow of a single phase, incompressible fluid in a porous medium with fractures. In this model, the flow obeys Forchheimer's law in the fracture and Darcy's law in the rock matrix.

MOTS-CLÉS : milieu poreux, fracture, loi de Forchheimer, décomposition de domaine.

KEYWORDS : porous media, fracture, Forchheimer's Law, domain decomposition.

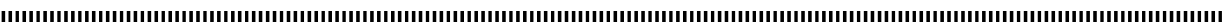

Volume 5 - 2006, pages 129-143 - ARIMA

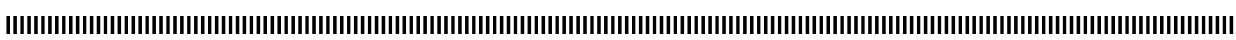

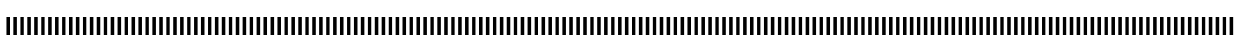

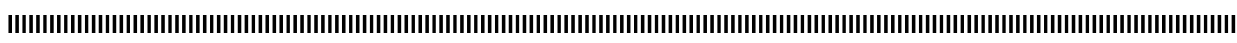

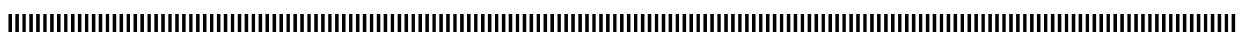

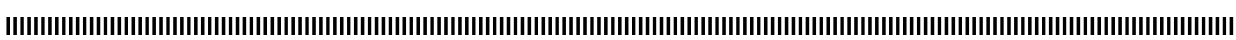

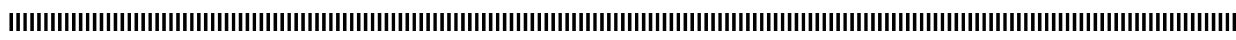
|

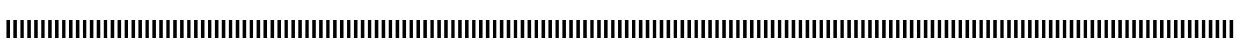

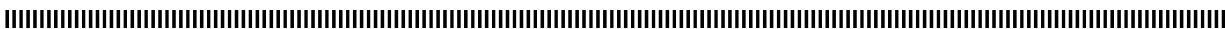




\section{Introduction}

De nombreux travaux de modélisation traitent de l'écoulement et du transport dans les milieux poreux fracturés. Ainsi le modèle double porosité est obtenu grâce à un processus d'homogénéisation. Il s'applique si la fracturation est suffisamment dense, régulière et si les fractures sont interconnectées, voir [6], [7]. Ce modèle prend bien en compte la présence des fractures et les échanges intervenant entre les fractures et la roche.

D'autres modèles représentent des réseaux de fractures sans prendre en compte ce qui se passe dans la roche. L'objectif est alors de vérifier si on peut représenter le réseau de fractures par un milieu poreux dont on peut calculer les perméabilités, voir par exemple [8].

Les travaux menés en [1] et [2] se sont intéressés aux grandes fractures ou failles, à perméabilité élevée. Ces fractures sont ramenées à des interfaces entre les milieux poreux qui les entourent. Ces interfaces sont mathématiquement particulières car si la pression reste continue à leur passage la composante normale du flux ne l'est pas. Ce modèle, obtenu via une analyse asymptotique, permet l'échange entre les fractures et la matrice comme le font les modèles double porosité. Un travail réalisé dans [13] et [14] a étendu le modèle précédent à des fractures de faible perméabilité dite fractures barrières. Pour ces fractures la condition de continuité de la pression à travers la fracture interface n'est plus valable. Un autre travail qui modélise les fractures comme des interfaces a été réalisé dans [16], mais le modèle ne traite pas des échanges entre la roche et les fractures de la même façon.

Dans cet article nous présentons un modèle numérique pour l'écoulement dans un milieu poreux avec une fracture dans laquelle l'écoulement suit la loi de Forchheimer. La loi de Forchheimer est une loi non-linéaire qui généralise la loi de Darcy. Elle est valable aussi bien quand l'écoulement est lent que rapide. Le modèle que nous décrivons ici est basé sur le modèle de [1] et [2]. Dans ce modèle la fracture est une fracture de grande perméabilité et elle est identifiée à une interface à travers laquelle la pression est continue. Dans ce qui suit nous décrivons notre modèle et nous présentons quelques expériences numériques pour le valider. Dans un autre article nous aborderons ses aspects mathématiques. Dans la section 2 de cet article nous explicitons les lois gouvernant l'écoulement dans un milieu poreux. Dans la section 3 le problème modèle que nous traitons est presenté. La section 4 est consacrée à la dérivation du modèle que nous avons développé à partir d'un modèle de référence. La discrétisation numérique du modèle développé fait alors l'objet de la section 5. Dans la section 6 nous donnons des résultats numériques et nous terminons avec une conclusion dans la section 7 . 


\section{2. Équations gouvernant l'écoulement dans un milieu poreux}

L'écoulement dans un domaine $\Omega$ représentant un milieu poreux est gouverné par une loi de conservation de masse qui s'écrit, en faisant l'hypothèse d'incompressibilité :

$$
\operatorname{div}(\mathbf{u})=q \text { dans } \Omega,
$$

où $q$ est un terme source et $\mathbf{u}$ la vitesse de Darcy. En négligeant la gravité, cette dernière est donnée par la loi de Darcy :

$$
\mathbf{u}+K \nabla p=0, \quad \text { dans } \Omega,
$$

où $p$ est la pression et $K$ la perméabilité du milieu.

Cette loi qui modèlise des phénomènes lents néglige les forces d'inertie devant les forces de viscosité. Pour des phénomènes où les vitesses d'écoulement sont élevées, la loi de Forchheimer devient plus adéquate pour la modélisation de l'écoulement. Elle tient compte d'un terme non linéaire qui traduit l'influence des forces d'inertie, voir [3], [5], [9], [12] et [17]. Elle s'écrit :

$$
G(\mathbf{u})+K \nabla p=0 \text { dans } \Omega .
$$

où $G$ est définie par $G(\mathbf{u})=(1+b|\mathbf{u}|) \mathbf{u}$, avec $b$ une constante d'inertie.

Nous allons présenter dans ce papier un modèle numérique d'écoulement pour un fluide monophasique incompressible dans un milieu poreux fracturé. L'écoulement dans la fracture est supposé assez élevé pour que les termes d'inerties ne soient pas négligés. Il est alors gouverné par la loi de Forchheimer. Dans les sous-domaines, les effets d'inertie sont négligeables et l'écoulement est gouverné par la loi de Darcy.

Dans le paragraphe suivant, nous présenterons la configuration que nous avons traitée ainsi que le modèle numérique que nous avons élaboré et qui nous permet de traiter la fracture comme une interface .

\section{Description du problème}

Soit $\Omega$ un domaine connexe de $\Omega \subset \mathbb{R}^{n}, n=2$ ou 3 , de frontière $\Gamma=\partial \Omega$. La fracture $\Omega_{f}$ est supposée être un sous-domaine de $\Omega$ d'ouverture égale à $d$, séparant deux autres sous-domaines connexes $\Omega_{1}$ et $\Omega_{2}$ (voir Figure 1). Nous avons :

$$
\Omega \backslash \bar{\Omega}_{f}=\Omega_{1} \cup \Omega_{2}, \quad \Omega_{1} \cap \Omega_{2}=\phi .
$$

Notons $\Gamma_{i}$ la partie de la frontière de $\Omega_{i}$ se trouvant sur la frontière de $\Omega, i=1,2, f$ : 


$$
\Gamma_{i}=\partial \Omega_{i} \cap \Gamma, \quad i=1,2, f,
$$

et $\gamma_{i}$ la partie de frontière de $\Omega_{i}$ à la frontière de la fracture $\Omega_{f}$ :

$$
\gamma_{i}=\partial \Omega_{i} \cap \partial \Omega_{f} \cap \Omega, \quad i=1,2 .
$$

Notons aussi par $\mathbf{n}$ un vecteur unitaire dans $\mathcal{R}^{n}$, orthogonal à $\gamma_{i}, i=1,2$, et dirigé vers l'extérieur de $\Omega_{1}$ et par $\eta$ un champ de vecteurs unitaires sur $\Gamma$ dirigé vers l'extérieur de $\Omega$.

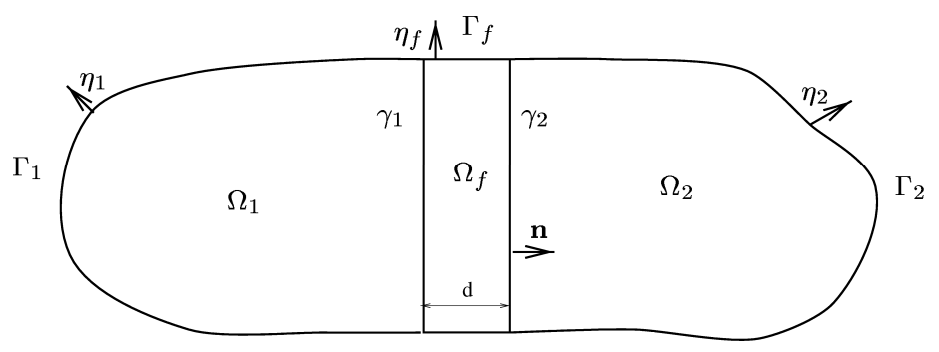

Figure 1. Le domaine $\Omega$ avec la fracture $\Omega_{f}$

Nous supposons que l'écoulement dans $\Omega$ est gouverné par l'équation de conservation de masse couplée avec une loi de comportement reliant le gradient de pression $p$ à la vitesse de l'écoulement $u$. Cette loi est la loi de Forchheimer dans la fracture $\Omega_{f}$ et la loi de Darcy dans les sous-domaines $\Omega_{1}$ et $\Omega_{2}$. Afin d'obtenir une écriture compacte de nos équations, nous écrivons la loi de Darcy comme la loi de Forchheimer [3] avec un terme d'inertie nul. Par simplicité nous assignons sur $\Gamma$ ' une condition de Dirichlet, et nos équations sur $\Omega$ s'écrivent alors :

$$
\begin{aligned}
\operatorname{div} \mathbf{u} & =q & & \operatorname{sur} \Omega \\
(1+b|\mathbf{u}|) \mathbf{u} & =-K \nabla p & & \operatorname{sur} \Omega \\
p & =p_{D} & & \operatorname{sur} \Gamma,
\end{aligned}
$$

où $q$ est le terme source et $p_{D}$ est la pression sur le bord $\Gamma$. Nous supposons que le coefficient de perméabilité $K$ (ou la conductivité hydraulique), est borné inférieurement et supérieurement par des constantes positives :

$$
0<K_{\min } \leq K \leq K_{\max }<\infty,
$$

et que le coefficient d'inertie $b$ est positif dans $\Omega_{f}$ et égal à 0 dans $\Omega_{1} \cup \Omega_{2}$.

En notant $p_{i}, \mathbf{u}_{i}, K_{i}$, et $q_{i}$ les restrictions de $p, \mathbf{u}, K$, et $q$ respectivement à $\Omega_{i}, i=$ $1,2, f$, le problème de transmission qui nous intéresse s'écrit : 


$$
\begin{aligned}
& \operatorname{div} \mathbf{u}_{i}=q_{i} \\
& \mathbf{u}_{i}=-K_{i} \nabla p_{i} \quad \text { dans } \Omega_{i}, \quad i=1,2, \\
& \left(1+b\left|\mathbf{u}_{f}\right|\right) \mathbf{u}_{f}=-K_{f} \nabla p_{f} \\
& p_{i}=0 \\
& p_{i}=p_{f} \\
& u_{i} \cdot \mathbf{n}=u_{f} \cdot \mathbf{n} \\
& \begin{array}{ll}
\text { dans } \Omega_{i}, & i=1,2, f, \\
\text { dans } \Omega_{i}, & i=1,2, \\
\operatorname{dans} \Omega_{f}, & \\
\operatorname{sur} \Gamma_{i}, & i=1,2, f, \\
\operatorname{sur} \gamma_{i}, & i=1,2, \\
\operatorname{sur} \gamma_{i}, & i=1,2 .
\end{array}
\end{aligned}
$$

où $K_{1}$ et $K_{2}$ sont les tenseurs de perméabilité dans $\Omega_{1}$ et $\Omega_{2}$ et $K_{f}$ le tenseur de perméabilité de Forchheimer dans $\Omega_{f}$.

Le système des équations [5] peut être résolu en utilisant des techniques de décomposition de domaine en considérant $\Omega_{f}$ comme un troisième sous-domaine. La technique proposée dans la suite est de ne pas traiter $\Omega_{f}$ comme un sous-domaine mais comme une interface $\gamma$ entre les sous-domaines $\Omega_{1}$ et $\Omega_{2}$ et en imposant des nouveaux conditions de transmission.

\section{Modélisation de la fracture}

Pour tenir compte de la taille de la fracture, très petite par rapport aux autres dimensions du domaine, une possibilité est d'utiliser un maillage très fin autour. Le modèle que nous proposons avec la technique de résolution que nous utilisons, nous permettront d'éviter d'avoir à le faire. Plus simple et moins coûteux, ce modèle est valable sous les hypothèses d'une ouverture très petite et une perméabilité grande de la fracture par rapport respectivement aux dimensions et la perméabilité de la matrice environnante. L'écoulement tangentiel est aussi considéré très élevé par rapport à l'écoulement normal. Dans ce nouveau modèle, la fracture $\Omega_{f}$ est remplacée par une interface $\gamma$ entre les deux sousdomaines $\Omega_{1}$ et $\Omega_{2}$ : voir Figure 2 .

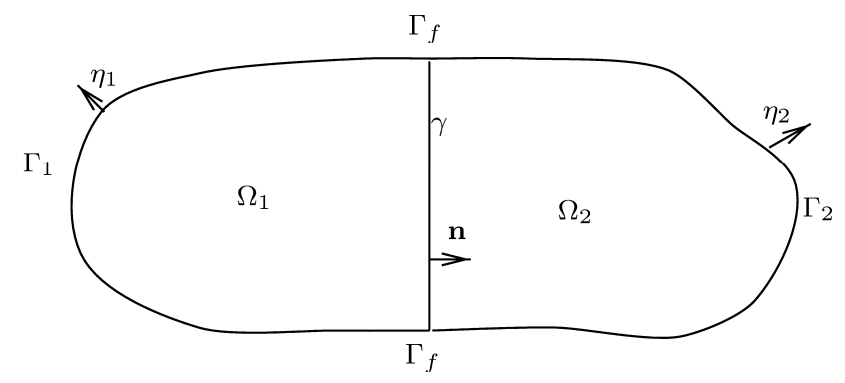

Figure 2. Le domaine $\Omega$ avec la fracture $\gamma$ comme interface 


\subsection{Intégration de l'équation de conservation de masse :}

Nous identifions $\Omega_{f}$ avec le produit $\gamma \times\left(-\frac{d}{2}, \frac{d}{2}\right)$. Pour obtenir les équations sur l'interface $\gamma$ nous décomposons le vecteur $\mathbf{u}_{f}$ en ses composantes normale et tangentielle à la fracture, $\mathbf{u}_{f, n}$ et $\mathbf{u}_{f, \tau}$, respectivement. Nous notons $\nabla_{\tau}$ et $\operatorname{div}_{\tau}$ le gradient tangentiel et la divergence tangentielle, $\nabla_{n}$ et $\operatorname{div}_{n}$ le gradient normal et la divergence normale.

Avec ces notations, la première équation du système [5] pour $i=f$, s'écrit :

$$
\operatorname{div}_{n} \mathbf{u}_{f}+\operatorname{div}_{\tau} \mathbf{u}_{f}=q_{f} \quad \text { dans } \quad \Omega_{f}
$$

Son intégration suivant la direction normale à la fracture sur un segment $\left(-\frac{d}{2}, \frac{d}{2}\right)$ aboutit à l'équation :

$$
\mathbf{u}_{f} \cdot \mathbf{n}_{\mid \gamma 2}-\mathbf{u}_{f} \cdot \mathbf{n}_{\mid \gamma 1}+\operatorname{div}_{\tau} \mathbf{U}_{f}=Q_{f} \quad \operatorname{sur} \quad \gamma
$$

où

$$
\mathbf{U}_{f}=\int_{-\frac{d}{2}}^{\frac{d}{2}} \mathbf{u}_{f, \tau} d n \quad \text { et } \quad Q_{f}=\int_{-\frac{d}{2}}^{\frac{d}{2}} q_{f} d n,
$$

et en utilisant le fait que le flux est continu à travers $\gamma_{1}$ et $\gamma_{2}$, on obtient :

$$
\operatorname{div}_{\tau} \mathbf{U}_{f}=Q_{f}+\left(\mathbf{u}_{1} \cdot \mathbf{n}_{\mid \gamma 1}-\mathbf{u}_{2} \cdot \mathbf{n}_{\mid \gamma 2}\right) \quad \text { sur } \quad \gamma .
$$

L'équation [8] ne fait qu'exprimer la conservation de la masse dans $\gamma$ avec un terme source supplémentaire $\mathbf{u}_{1} \cdot \mathbf{n}_{\mid \gamma 1}-\mathbf{u}_{2} \cdot \mathbf{n}_{\mid \gamma 2}$ tenant compte de l'échange entre $\Omega_{f}$ (devenue $\gamma$ dans le nouveau modèle) et les sous-domaines $\Omega_{1}$ et $\Omega_{2}$.

\subsection{Intégration de la loi de comportement :}

Avant de procéder à l'intégration des équations suivant la direction normale à la fracture, faisons l'hypothèse que l'écoulement dans $\Omega_{f}$ est gouverné par la loi de Forchheimer dans la direction tangentielle et par la loi de Darcy dans la direction normale. Cette hypothèse est justifiée car le rapport entre la largeur et la longueur de la fracture est petit, rendant les effets d'inertie plus importants dans la sens de la longueur. La décomposition de la deuxième équation du système [5] suivant la direction tangentielle et la direction normale s'écrit :

$$
\begin{aligned}
\left(1+b\left|\mathbf{u}_{f}\right|\right) \mathbf{u}_{f, \tau} & =-K_{f} \nabla_{\tau} p_{f} \\
\mathbf{u}_{f, n} & =-K_{f} \nabla_{n} p_{f} .
\end{aligned}
$$

L'intégration de la première équation du système [9] sur un segment $\left(-\frac{d}{2}, \frac{d}{2}\right)$, s'écrit alors :

$$
\int_{-\frac{d}{2}}^{\frac{d}{2}}\left(1+b\left|\mathbf{u}_{f}\right|\right) \mathbf{u}_{f, \tau} d n=-K_{f} \nabla_{\tau} \int_{-\frac{d}{2}}^{\frac{d}{2}} p_{f} d n
$$


et en approchant $\left|\mathbf{u}_{f}\right|$ par :

$$
\left|\mathbf{u}_{f}\right|=\left|\mathbf{u}_{f, \tau}+\mathbf{u}_{f, n}\right| \simeq\left|\mathbf{u}_{f, \tau}\right|\left(1+\frac{1}{2} \frac{\mathbf{u}_{f, n}^{2}}{\mathbf{u}_{f, \tau}^{2}}\right)
$$

puis en négligeant $\mathbf{u}_{f, n}$ devant $\mathbf{u}_{f, \tau}$ (i.e : $\quad \frac{\mathbf{u}_{f, n}^{2}}{\mathbf{u}_{f, \tau}^{2}} \ll 1$ ), on obtient :

$$
\left|\mathbf{u}_{f}\right| \simeq\left|\mathbf{u}_{f, \tau}\right| \simeq \frac{\left|\mathbf{U}_{f}\right|}{d} .
$$

L'injection de cette dernière approximation dans [10] nous donne la nouvelle loi de Forchheimer qui gouverne l'écoulement dans $\gamma$ :

$$
\left(1+\frac{b}{d}\left|\mathbf{U}_{f}\right|\right) \int_{-\frac{d}{2}}^{\frac{d}{2}} \mathbf{u}_{f, \tau} d n=-K_{f} d \nabla_{\tau} P_{f}
$$

où $P_{f}=\frac{1}{d} \int_{-\frac{d}{2}}^{\frac{d}{2}} p_{f, \tau} d n$ et où nous avons supposé que $K_{f}$ est constante sur l'intervalle $\{s\} \times\left(-\frac{d}{2}, \frac{d}{2}\right), \forall s \in \gamma$. Une écriture équivalente à [11] est :

$$
\left(1+\frac{b}{d}\left|\mathbf{U}_{f}\right|\right) \mathbf{U}_{f}=-K_{f} d \nabla_{\tau} P_{f} .
$$

En ce qui concerne la deuxième équation du système [9], sous l'hypothèse que $\mathbf{u}_{f, n}$ est petit et $K_{f}$ est grand, nous supposons que la pression $p$ est continue à travers $\gamma$ :

$$
p_{1 \mid \gamma}=P_{f}=p_{2 \mid \gamma} .
$$

Après intégration des deux équations qui modélisent l'écoulement dans la fracture, le nouveau système d'équations à résoudre devient :

$$
\begin{aligned}
\operatorname{div} \mathbf{u}_{i} & =q_{i} & & \operatorname{dans} \Omega_{i}, \quad i=1,2 \\
\mathbf{u}_{i} & =-K_{i} \nabla p_{i} & & \operatorname{dans} \Omega_{i}, \quad i=1,2 \\
\operatorname{div}_{\tau} \mathbf{U}_{f} & =Q_{f}+\left(\mathbf{u}_{1} \cdot \mathbf{n}-\mathbf{u}_{2} \cdot \mathbf{n}\right) & & \operatorname{sur} \gamma, \\
\left(1+\frac{b}{d}\left|\mathbf{U}_{f}\right|\right) \mathbf{U}_{f} & =-K_{f} d \nabla_{\tau} P_{f} & & \operatorname{sur} \gamma, \\
p_{i} & =p_{D} & & \operatorname{sur} \Gamma_{i}, \quad i=1,2 \\
p_{i} & =P_{f} & & \operatorname{sur} \gamma, \quad i=1,2 \\
P_{f} & =P_{f_{D}} & & \text { sur } \partial \gamma,
\end{aligned}
$$

où les équations sur $\Omega_{f}$ dans [5] ont été remplacées par les équations [8] et [12] et les données de Dirichlet $P_{f_{D}}$ sur la bord de $\gamma$ sont obtenues en faisant la moyenne sur les intervalles $\left(-\frac{d}{2}, \frac{d}{2}\right)$ dans $\partial \gamma \times\left(-\frac{d}{2}, \frac{d}{2}\right)=\Gamma_{f}: P_{f_{D}}=\frac{1}{d} \int_{-\frac{d}{2}}^{\frac{d}{2}} p_{D} d n$. 
Remarque : La prise en compte du terme d'inertie du à un écoulement rapide dans la fracture se justifie quand la perméabilité $K_{f}$ est une quantité scalaire élevée. Mais il y a des fractures où $K_{f}$ est un tenseur avec une perméabilité tangentielle $K_{f, \tau}$ élevée et une perméabilité normale $K_{f . n}$ petite. Dans ce cas, et même si on peut continuer à considérer que l'écoulement suit la loi de Forchheimer dans le sens tangentiel, l'hypothèse $p_{1}=p_{2}=P_{f}$ n'est plus appropriée. On intègre alors la deuxième équation de [13] à travers la fracture pour obtenir à la place $p_{1}-p_{2}=\frac{d}{2 K_{f, n}}\left(\mathbf{u}_{1} \cdot \mathbf{n}+\mathbf{u}_{2} \cdot \mathbf{n}\right)$ et on utilise cette dernière équation avec $p_{1}+p_{2}=2 P_{f}$. Un modèle avec une telle perméabilité dans la fracture mais avec un flux de Darcy a été développé dans [14]. Nous poursuivrons cette démarche avec un écoulement de Forchheimer dans la fracture dans un article à venir.

\section{Discrétisation Numérique}

Pour calculer une solution approchée du système [13] nous utilisons les éléments finis mixtes. Nous commençons avec une triangulation $\mathcal{T}_{h}$ du domaine $\Omega_{\gamma}=\Omega_{1} \cup \Omega_{2} \cup \gamma$ qui est telle que chaque élément de la triangulation est inclu soit dans $\Omega_{1}$, soit dans $\Omega_{2}$. Nous avons approché $p_{i}$ et $\mathbf{u}_{i}$ dans l'espace de fonctions constantes par élément, $M_{h, i}$, et l'espace de Raviart-Thomas-Nédelec d'ordre le plus bas, $W_{h, i}$, respectivement, $i=1,2$.

La fracture $\gamma$ hérite d'un maillage $\mathcal{T}_{f, h}$, et nous approchons $P_{f}$ et $\mathbf{U}_{f}$ aussi dans l'espace de fonctions constantes par élément, $M_{h, f}$, et l'espace de Raviart-Thomas-Nedelec d'ordre le plus bas, $W_{h, f}$, respectivement, mais en dimension $n-1$.

De la façon habituelle pour les éléments finis mixtes, voir [4] ou [10], on divise les deuxième et quatrième équations dans $\left(\mathcal{P}_{h}\right)$ par les coefficients de perméabilité, on les multiplie par les fonctions tests appartenant aux espaces d'approximations et on les intégre sur les domaines $\Omega_{i}$ ou $\gamma$.

Après intégration par partie des termes contenant un gradient ou un gradient tangentiel, on aboutit au problème discret qui consiste à trouver

$\left(\left(\mathbf{u}_{h, 1}, \mathbf{u}_{h, 2}, \mathbf{U}_{h, f}\right),\left(p_{h, 1}, p_{h, 2}, P_{h, f}\right)\right) \in\left(W_{h, 1} \times W_{h, 2} \times W_{h, f}\right) \times\left(M_{h, 1} \times M_{h, 2} \times M_{h, f}\right)$ tels que pour tout 
$\left(\left(\mathbf{v}_{h, 1}, \mathbf{v}_{h, 2}, \mathbf{v}_{h, f}\right),\left(r_{h, 1}, r_{h, 2}, r_{h, f}\right)\right) \in\left(W_{h, 1} \times W_{h, 2} \times W_{h, f}\right) \times\left(M_{h, 1} \times M_{h, 2} \times M_{h, f}\right)$ on a :

$\left(\mathcal{P}_{h}\right)$

$$
\begin{aligned}
& \int_{\Omega_{i}} K_{i}^{-1} \mathbf{u}_{h, i} \cdot \mathbf{v}_{h, i}-\int_{\Omega_{i}} p_{h, i} \operatorname{div} \mathbf{v}_{h, i}=-\int_{\gamma} P_{h, f} \mathbf{v}_{h, i} \cdot \mathbf{n}_{i}-\int_{\Gamma_{i}} p_{D} \mathbf{v}_{h, i} \cdot \eta_{i}, i=1,2 \\
& \int_{\Omega_{i}} \operatorname{div} \mathbf{u}_{h, i} r_{h, i}=\int_{\Omega_{i}} q_{i} r_{h, i}, \quad i=1,2 \\
& \int_{\gamma}\left(K_{f} d\right)^{-1}\left(1+\frac{b}{d}\left|\mathbf{U}_{h, f}\right|\right) \mathbf{U}_{h, f} \cdot \mathbf{v}_{h, f}-\int_{\gamma} P_{h, f} \operatorname{div} \mathbf{v}_{h, f}=-\int_{\gamma \cap \Gamma} p_{D} \mathbf{v}_{h, f} \cdot \eta_{f} \\
& \int_{\gamma} \operatorname{div}_{\tau} \mathbf{U}_{h, f} r_{h, f}=\int_{\gamma} Q_{f} r_{h, f}+\int_{\gamma}\left(\mathbf{u}_{h 1} \cdot \mathbf{n}-\mathbf{u}_{h, 2} \cdot \mathbf{n}\right) r_{h, f},
\end{aligned}
$$

où $\mathbf{n}_{1}=\mathbf{n}=-\mathbf{n}_{2}$ et $\eta_{i}=\eta_{\mid \Gamma_{i}}, i=1,2, f$.

Pour résoudre ce problème, on a utilisé les techniques de décomposition de domaine, voir [15]. On procède d'abord par l'élimination des inconnues dans $M_{h, i}$ et $W_{h, i}, i=$ 1,2 , avec l'aide des operateurs de Steklov-Poincaré. Pour cela, on définit pour chaque $i$, l'operateur de Steklov-Poincaré :

$$
\begin{aligned}
\mathcal{S}_{i}: M_{h, f} & \longrightarrow M_{h, f} \\
r_{h, f} & \mapsto-\mathbf{u}_{h, i}^{0} \cdot \mathbf{n}_{i}
\end{aligned}
$$

qui associe à un élément $r_{h, f} \in M_{h, f}$ l'élément $-\mathbf{u}_{h, i}^{0} \cdot \mathbf{n}_{i} \in M_{h, f}$ où $\left(\mathbf{u}_{i}^{0}, p_{h, i}^{0}\right) \in$ $W_{h, i} \times M_{h, i}$ est la solution du problème :

$$
\begin{gathered}
\text { Trouver }\left(\mathbf{u}_{h, i}^{0}, p_{h, i}^{0}\right) \in W_{h, i} \times M_{h, i} \text { telle que } \\
\left(\mathcal{P}_{i}^{0}\right) \int_{\Omega_{i}} K_{i}^{-1} \mathbf{u}_{h, i}^{0} \cdot \mathbf{v}_{h, i}-\int_{\Omega_{i}} p_{h, i}^{0} \operatorname{div}_{h, i}=-\int_{\gamma} r_{h, f} \mathbf{v}_{h, i} \cdot \mathbf{n}_{i} \quad \forall \mathbf{v}_{h, i} \in W_{h, i} \\
\int_{\Omega_{i}}^{\operatorname{div} \mathbf{u}_{h, i}^{0} r_{h, i}=0 \quad \forall r_{h, i} \in M_{h, i} .}
\end{gathered}
$$

Nous avons besoin aussi de $\chi_{i}=\mathbf{u}_{h, i}^{*} \cdot \mathbf{n}_{i} i=1,2$ où $\left(\mathbf{u}_{h, i}^{*}, p_{h, i}^{*}\right) \in W_{h, i} \times M_{h, i}$ est la solution du problème

$$
\begin{aligned}
& \begin{array}{l}
\text { Trouver }\left(\mathbf{u}_{h, i}^{*}, p_{h, i}^{*}\right) \in W_{h, i} \times M_{h, i} \text { telle que } \\
\left(\mathcal{P}_{i}^{*}\right) \int_{\Omega_{i}} K_{i}^{-1} \mathbf{u}_{h, i}^{*} \cdot \mathbf{v}_{h, i}-\int_{\Omega_{i}} p_{h, i}^{*} \operatorname{div} \mathbf{v}_{h, i}=-\int_{\Gamma_{i}} p_{D} \mathbf{v}_{h, i} \cdot \eta_{i} \quad \forall \mathbf{v}_{h, i} \in W_{h, i}
\end{array} \\
& \int_{\Omega_{i}} \operatorname{div} \mathbf{u}_{h, i}^{*} r_{h, i}=\int_{\Omega_{i}} q_{i} r_{h, i} \quad \forall r_{h, i} \in M_{h, i} .
\end{aligned}
$$

On remarque que si $\left(\left(\mathbf{u}_{h, 1}, \mathbf{u}_{h, 2}, \mathbf{U}_{h, f}\right),\left(p_{h, 1}, p_{h, 2}, P_{h, f}\right)\right)$ est la solution de $\left(\mathcal{P}_{h}\right)$ et $\left(\mathbf{u}_{h, i}^{0}, p_{h, i}^{0}\right)$ est la solution de $\left(\mathcal{P}_{i}^{0}\right)$ avec $r_{h, f}=P_{h, f}$ alors

$$
p_{h, i}=p_{h, i}^{0}+p_{h, i}^{*} \quad \text { et } \quad \mathbf{u}_{h, i}=\mathbf{u}_{h, i}^{0}+\mathbf{u}_{h, i}^{*} .
$$


Donc, en écrivant $\mathbf{u}_{h, i} \cdot \mathbf{n}_{i}=\mathcal{S}_{i}\left(P_{h, f}\right)+\chi_{i}$, on obtient $\mathbf{U}_{h, f}$ et $P_{h, f}$ en résolvant le problème suivant :

$$
\begin{gathered}
\text { Trouver }\left(\mathbf{U}_{h, f}, P_{h, f}\right) \in W_{h, f} \times M_{h, f} \text { telle que } \forall\left(\mathbf{v}_{h, f}, r_{h, f}\right) \in W_{h, f} \times M_{h, f}, \\
\left(\mathcal{P}_{\gamma}\right) \int_{\gamma} K_{f}^{-1}\left(1+\frac{b}{d}\left|\mathbf{U}_{h, f}\right|\right) \mathbf{U}_{h, f} \cdot \mathbf{v}_{h, f}-\int_{\gamma} P_{h, f} \operatorname{divv}_{h, f}=-\int_{\partial \gamma} p_{D} \mathbf{v}_{h, f} \cdot \eta_{f} \\
\int_{\gamma} \operatorname{div}_{\tau} \mathbf{U}_{h, f} r_{h, f}+\int_{\gamma}\left(\mathcal{S}_{1}\left(P_{h, f}\right)+\mathcal{S}_{2}\left(P_{h, f}\right)\right) r_{h, f}=\int_{\gamma} Q_{f} r_{h, f}+\int_{\gamma}\left(\chi_{1}+\chi_{2}\right) r_{h, f} .
\end{gathered}
$$

On peut ensuite obtenir $\mathbf{u}_{h, i}$ et $p_{h, i}, i=1,2$, avec l'aide de [14].

Pour résoudre le problème non-linéaire $\left(\mathcal{P}_{\gamma}\right)$, nous avons utilisé deux méthodes différentes : la méthode de quasi-Newton et la méthode de point fixe.

\section{Résultats numériques}

Nous avons faits des expériences numériques en deux dimensions pour un problème modèle simple : le domaine $\Omega$ est un rectangle de dimension 2.01 par 1 . Il est formé de deux sous-domaines carrés $\Omega_{1}$ et $\Omega_{2}$ de côte 1 , séparés par une fracture de largeur $d=0.01$ qui traverse le domaine : voir Figure 3. La perméabilité dans les sous-domaines $\Omega_{1}$ et $\Omega_{2}$ est supposée constante et égale à $10^{-9}$ et la perméabilité dans la fracture est $10^{-6}$. Le coefficient de Forchheimer $b$ est égal à 10 dans la fracture et nul dans les deux sous domaines $\Omega_{1}$ et $\Omega_{2}$ où l'écoulement suit la loi de Darcy. Les bords nord et sud des sous-domaines sont supposés imperméables (condition de Neumann homogène). Une pression de $10^{8}$ est imposée en haut de la fracture et sur le bord droit du domaine $\Omega$ tandis que la pression en bas de la fracture et sur le bord gauche de $\Omega$ est supposé nulle.

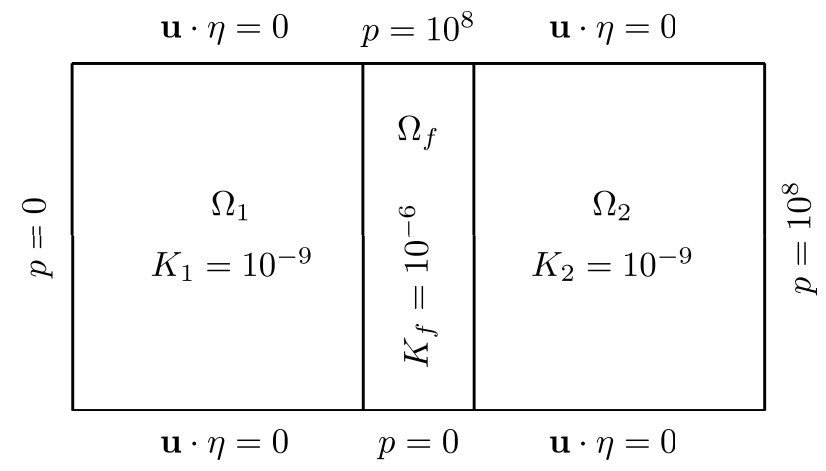

Figure 3.

Cas-test 
Sur les figures 4 et 5 nous présentons les résultats obtenus avec le modèle interface et ceux obtenus en utilisant le modèle de référence, c'est à dire le modèle où la fracture est bidimensionnelle. Dans les deux cas, nous avons utilisé un maillage $20 \times 20$ pour chacun des sous-domaines $\Omega_{1}$ et $\Omega_{2}$, et aussi pour la fracture $\Omega_{f}$ dans le cas du modèle de référence.
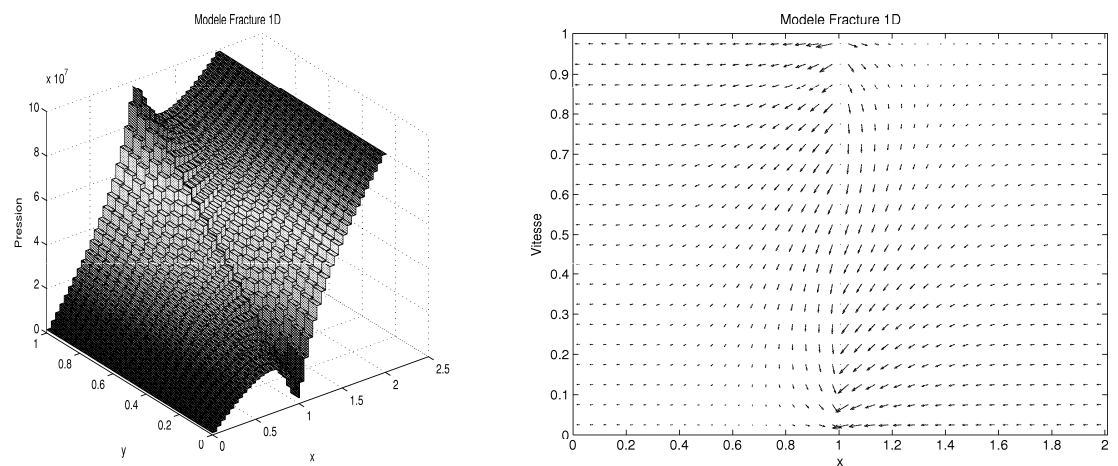

Figure 4. Pression et vitesse obtenus via le modèle interface
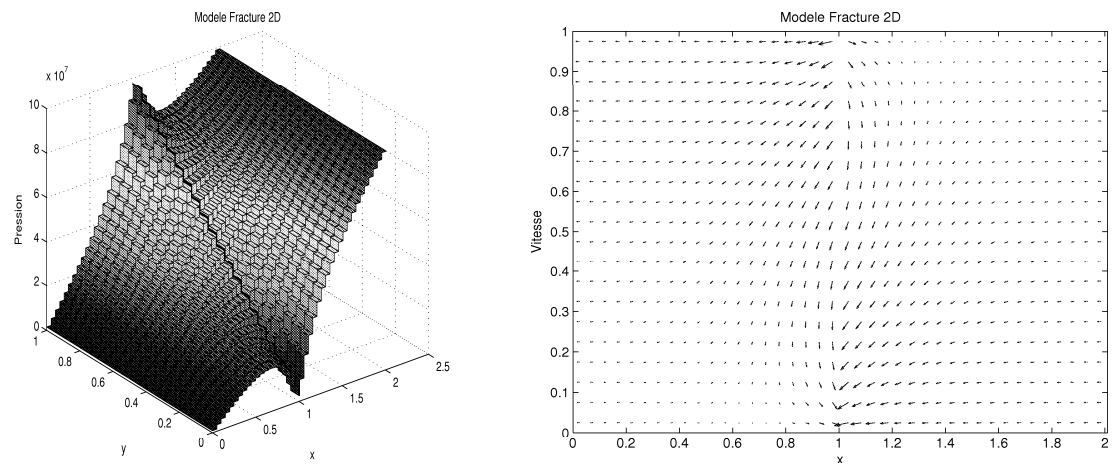

Figure 5. Pression et vitesse obtenus via le modèle de référence

Nous observons la bonne concordance entre les résultats obtenus par les deux modèles interface et référence, aussi bien pour la pression que pour la vitesse. 
Pour la résolution des deux problèmes, celui de référence et celui avec interface, nous avons utilisé la méthode des éléments finis mixtes avec des techniques de décomposition de domaine afin de ramener le problème à un problème posé dans la fracture. Pour le modèle de référence, le problème obtenu est non-linéaire bidimensionnel : l'interaction avec les sous-domaines $\Omega_{1}$ et $\Omega_{2}$ s'effectue par l'intermédiaire des conditions de Neumann sur les bords gauche et droite de la fracture $\Omega_{f}$. Pour le problème avec interface le problème obtenu est un problème non-linéaire unidimensionnel où l'interaction avec les sous-domaines $\Omega_{1}$ et $\Omega_{2}$ s'effectue par l'intermédiaire d'un terme source. Pour la résolution de l'équation de Forchheimer, on a étudié deux méthodes itératives : la méthode de point fixe et une méthode de quasi-Newton. Le nombre d'iterations et le temps de calcul pour les deux méthodes et pour les deux modèles sont donnés dans le tableau suivant :

\begin{tabular}{|c|c|c|c|c|}
\hline Méthode & \multicolumn{2}{|c|}{ Modèle de référence } & \multicolumn{2}{c|}{ Modèle interface } \\
\hline \hline & $\begin{array}{c}\text { Nombre } \\
\text { d'itérations }\end{array}$ & $\begin{array}{c}\text { Temps } \\
\text { de calcul }\end{array}$ & $\begin{array}{c}\text { Nombre } \\
\text { d'itérations }\end{array}$ & $\begin{array}{c}\text { Temps } \\
\text { de calcul }\end{array}$ \\
\hline Point fixe & 84 & 52.950 & 78 & 1.3142 \\
\hline Quasi-Newton & 27 & $3.8489 \times 10^{4}$ & 37 & 1.8056 \\
\hline
\end{tabular}

Le gain important en temps de calcul avec le modèle interface est ainsi mis en évidence.

Nous attirons l'attention du lecteur sur le fait que les données choisies correspondent à une situation extrême de façon à mettre en évidence la différence entre le modèle de Forchheimer et celui de Darcy. On montre cette différence sur la figure 6 qui représente à gauche la vitesse tangentielle au centre de la fracture calculée avec le modèle de référence, et à droite la vitesse (tangentielle) dans la fracture calculée avec le modèle interface. On observe de nouveau la concordance entre les deux modèles (de référence et interface), et d'autre part l'éffet inertiel du modèle de Forchheimer par rapport à celui de Darcy (vitesses plus faibles pour Forchheimer). 

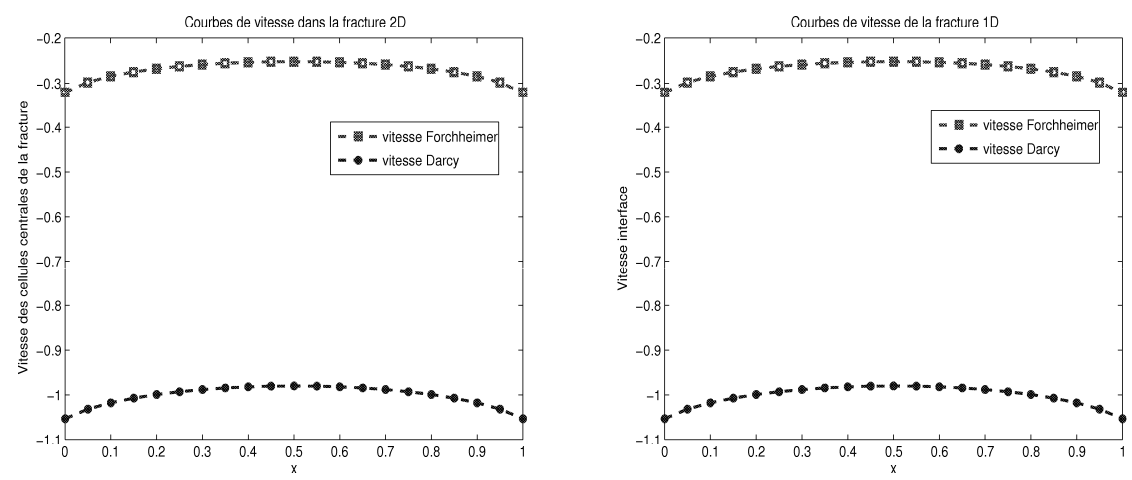

Figure 6. Vitesses dans la fracture calculées avec le modèle de référence (à gauche) et avec le modèle interface (à droite).

\section{Conclusion}

La présence de fractures de différentes natures dans les milieux poreux peut influencer l'écoulement du fluide d'une manière importante. Pour cela différents modèles ont été élaborés pour tenir compte de ces discontinuités géologiques.

Ici nous avons étendu le modèle presenté dans [1,2] au cas où l'écoulement dans la fracture est décrit par la loi de Forchheimer. Le modèle numérique présenté traite la fracture comme une interface. La méthode d'éléments finis mixtes est utilisée pour la discrétisation numérique. Des techniques de décomposition de domaines sont utilisées pour obtenir un problème non-linéaire à résoudre posé seulement sur l'interface. Ce modèle est valable seulement pour des fractures qui sont assez étroites pour qu'on puisse supposer que le terme d'inertie dans la direction normale à la fracture est négligeable, et qui sont assez perméables pour que la pression puisse être considerée continue à travers la fracture. Ceci n'est évidement pas le cas pour tous les problèmes où le loi de Forchheimer est appropriée. Mais pour les cas où ces hypothèses sont satisfaites, les premières indications sont que le modèle apporte des résultats satisfaisants et que le gain en temps de calcul est significatif.

Nous poursuivrons ce travail sur le plan théorique en faisant une analyse des modèles continu et discret, et sur le plan numérique en développant un code pour un modèle tridimensionnel où le gain en temps de calcul devrait être encore plus important. Nous 
envisageons aussi de traiter le cas des fractures qui s'intersectent.

\section{Remerciements}

Ce travail a été soutenu partiellement par le Ministère de la Recherche Scientifique, de la Technologie et du Développement des Compétences (MRSTDC, Tunisie) dans le cadre du programme LAB-STI-02, par le CMCU et l'équipe associée Inria-Lamsin MODESS.

\section{Bibliographie}

[1] C. Alboin.- Deux outils mathématiques pour modéliser l'écoulement et le transport de polluants dans une milieu poreux fracturé, Thése en mathématique appliquée. Université Paris IX Dauphine (soutenue le 13 janvier 2000).

[2] C. Alboin, J. Jaffré et J. E. Roberts. - Domain decomposition for flow in fractured porous media. - In : Domain Decomposition Methods in Sciences and Engineering, Domain Decomposition Press, pp. 365-373, - Bergen, 1999.

[3] J.L. Auriault, C. Geindreau et P. Royer - Lois d'écoulement en milieux poreux. Site internet : http :www.3s.hmg.inpg.fr/mme/Fr/Thèmes de recherche / Milieux Naturels / Lois d'ècoulement. Principales Publications, Juillet 2002.

[4] F. Brezzi et M. Fortin. - Mixed and Hybrid Finite Element Methods, Springer-Verlag, Berlin, 1991.

[5] J. Douglas, P. J. S. Paes Leme et T. Giorgi -Generalized Forchheimer flow in porous media, in Boundary Value Problems for Partial Differential Equations and Applications, Masson, Paris, In : Research Notes in Applied Mathematics, J.-L. Lions and C. Baiocchi (eds.), 29 (1993) 99113.

[6] J. Douglas, Jr. et T. Arbogast Dual porosity models for flow in naturally fractured reservoirs, In : in Dynamics of Fluids in Hierarchial Porous Formations, J. H. Cushman (ed.), Academic Press, 1990, pp. 177-221.

[7] J. Douglas, T. Arbogast et U. Hornung Derivation of the double porosity model of single phase flow via homogenization theory, In : SIAM J. Math. Anal., 1990, 21 823-836.

[8] J. Erhel et J.R. Dreuzy - Efficient algorithms for the determination of the connected fracture network and the solution of the steady-state flow equation in fracture networks,- In: Computers and Geosciences, 2002, 29 (1), pp. 107-111.

[9] J. Geertsma - Estimating the coefficient of inertial resistance in fluid flow through porous media. In : SPE J, 1974, 14(5), pp. 445-450.

[10] J. E. Roberts et J.-M. Thomas - Mixed and hybrid methods In : Handbook of Numerical Analysis, P. G. Ciarlet et J.-L. Lions, (eds) 2, Elesevier Science Publishers B. V. (North Holland), 1991, pp. 523-639.. Domain Decomposition Methods for partial differential equations. Claderon Press, Oxford, 1999. 
[11] M. Panfilov, M. Fourar - Physical splitting of nonlinear effects in high-velocity stable flow through porous media. In : Advanced in water resourses, available online at www.sciencedirect.com, 2006, 29, pp. 30-41.

[12] AE. Scheidegger - The physics of flow through porous media. New York : Macmillan, 1960.

[13] V. Martin - Simulations multidomaines des écoulements en milieu poreux. Thése en mathématique appliquée. Université Paris IX Dauphine (soutenue le 18 mars 2004).

[14] V. Martin, J. Jaffré et J. E. Roberts - Modeling fractures and barriers as interfaces for flow in porous media. In : SIAM Journal on Scientific Computing,2005, 26 pp. 1667-1691.

[15] A. Quateroni et A. Valli - Domain Decomposition Methods for partial differential equations. Claderon Press, Oxford, 1999.

[16] V. Reichenberger, H. Jakobs, P Bastian et R. Helmig - A mixed dimensional finite volume method for multiphase flow in fractured porous media submitted.

[17] S. Witaker The Forchheimer equation : theoretical development. Transport Pourous media 1996, 25, pp 27-61. 